\title{
The $25^{\text {th }}$ Scientific Conference for
}

\section{Information Systems and Computer Technology}

TOWARDS DIGITAL

TRANSFORMATION FOR

INTELLIGENCE OF MODERN BUSINESS ENTERPRISES

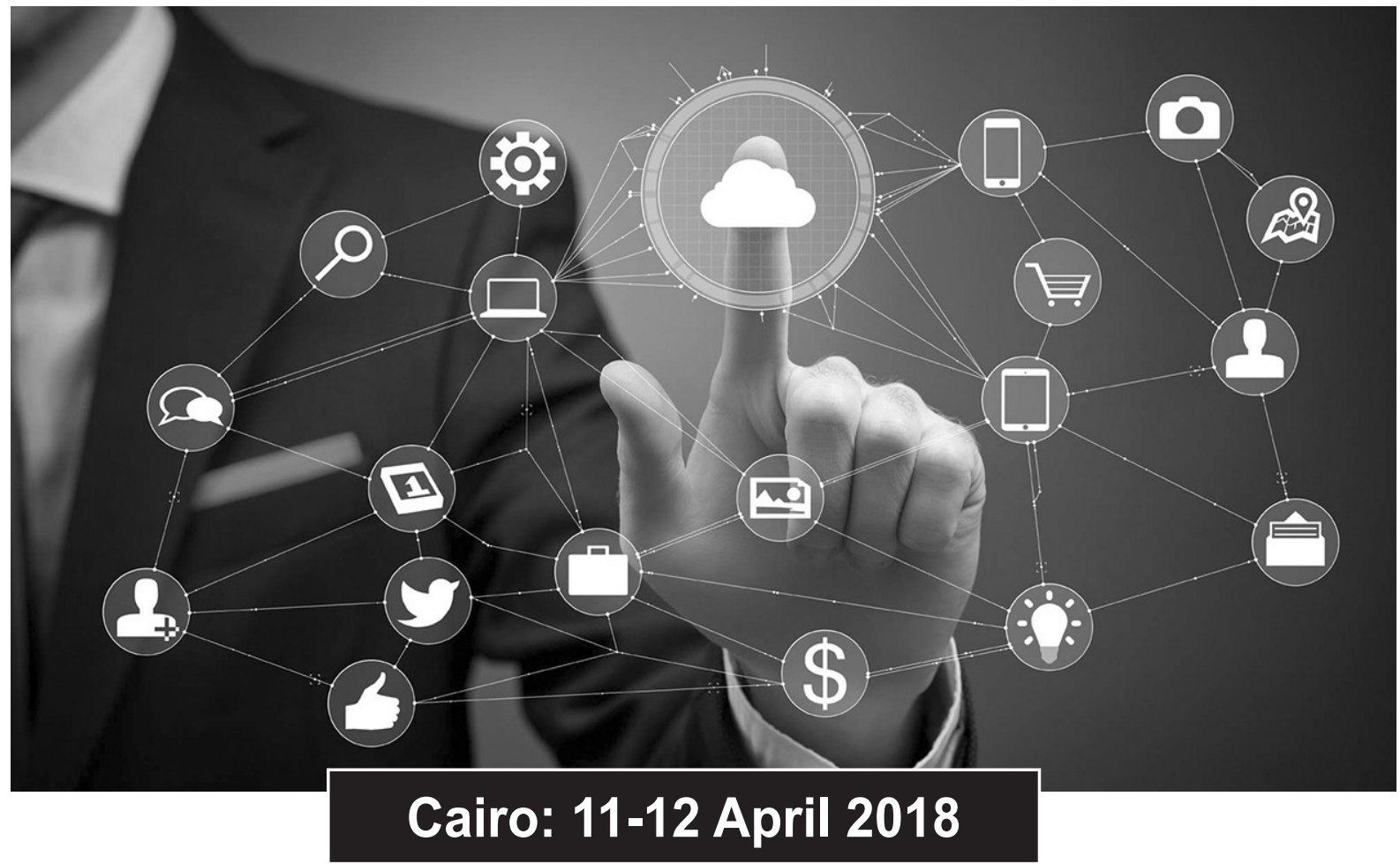

\section{Introduction}

Due to the emerging widespread of the digital transformation era and the new phenomenon of big data analytics, a new breed of modern business enterprises, intelligence for business service analytics is becoming a common fact to apply. Recently, many organizations have begun the shift to modern business intelligence and analytics, they are moving away from IT-centric reporting platform to modern BI \& Analytics platform according to Gartnerss 2016 Business Intelligence Magic Quadrant. Therefore, business intelligence and analytics has emerged as an important area reflecting the magnitude and impact of data-related problems to be solved in the contemporary 
digital transformation age. It is being noticed that the efforts and time consumed in affording to rely on reports that took considerable time to build and required technical skill to create and understand has shifted to the recent digital era that constitutes huge amounts and varied types of structured and unstructured data that poor into today business from every imangible sources. In the meantime, the present users are working from virtually everywhere; therefore they are connected with each other and with everything. So, they expect to have access to whatever data they need, when they require it using any device and software they prefer. In such a dynamic environment, today business users need to be able to explore the data for discovering new information and answer their queries, not just about what happened but also about why it happened. Therefore, Business Intelligence and Analytics solutions can only display what has already happened through reports and dashboards, as well as to gain advantages of the analytics within the era of digitalization, i.e., the new breed of business intelligence and analytics allow business users to quickly and easily investigate the data to find out why something happened, to uncover the root causes behind what, and only with sophisticated relevant visualization techniques that are easy to use and understand can business users get fact-based answers they need to create for real business advantages.

Too often organizations focus upon technology as a means of enhancing user perspective and factbased decision-making. And, while technology is an important enabler, it's a relatively small part of the equation. Instead, we know that the essential ingredients for success lie with people, process and organization - enabled by technology. We've long believed that there's a need for a fresh inperson forum for business and IT leaders to help achieve success with information, business enterprises intelligence and analytics.

Business enterprises, intelligence usually refers to the information that is available for the enterprise to make decisions on. A data warehousing (or data mart) system is the backend, or the infrastructural, component for achieving business intelligence. Business intelligence also includes the insight gained from doing data mining analysis, as well as unstructured data (thus the need for content management systems). For our purposes here, we will discuss business intelligence in the context of using a data warehouse infrastructure. This modern era of digital transformation is shaping the modern business enterprises, intelligence and analytics nowadays. As usual for the ESISACT's previous 24 conferences, this 25th Scientific Conference is also a unique event focusing upon strategies for success with business enterprises, intelligence and analytics for our today businesses.

\section{Conference Objectives:}

The 25th Scientific Conference on Towards Digital Transformation for Intelligence of Modern Business Enterprises aims to bring together leading academic scientists, researchers, scholar, and experts to exchange and share their experiences and research results on all aspects of Business Enterprises, Intelligence and Analytics, within the Digital transformation era. It also provides a premier interdisciplinary platform for researchers, practitioners and educators to present and discuss the most recent innovations, trends, and concerns as well as practical challenges encountered and solutions adopted in the fields of Business Intelligence and Analytics in the new era of digital revolution.

\section{Conference Themes:}

Key topics and highlights from the event:

- Digital Transformation Age,

- Business enterprises and digital transformation,

- Business enterprises, intelligencess Concepts, components, techniques and benefits,

- Business intelligence and business applications such as ERP, CRM, etc.

- Business Process Management and maturity, 
- Approaches to developing and building business enterprises, intelligence and Analytics,

- Critical capabilities of Business enterprises, intelligence $\&$ analytics platforms,

- Maximizing business enterprises, intelligence strategy and building up business capability,

- Business enterprises, intelligence and data-

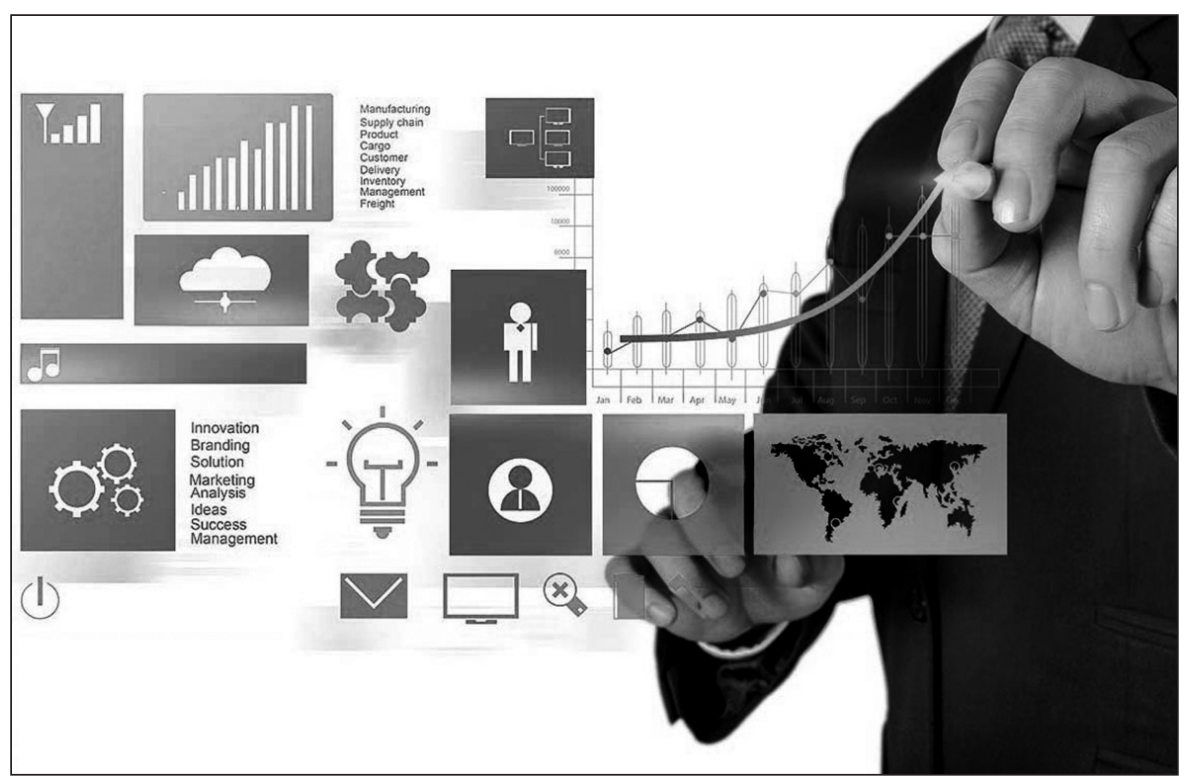
driven decision making,

- Business and Systems Engineering

- Security and risk with business enterprises intelligence

- etc.

\section{Call for Contributions:}

All interested researchers and practitioners are kindly encouraged to contribute to and help shape the conference through submissions of their research abstracts, papers and posters. Also, high quality research contributions describing original and unpublished results of conceptual, constructive, empirical, experimental, or theoretical work in all areas of Business Intelligence, Analytics, and digital transformation are cordially invited for presentation at the conference. The conference solicits contributions of abstracts, papers and posters that address themes and topics of the conference, including figures, tables and references of novel research materials.

Papers' submissions for the 25th. Scientific Conference are to be made in the following categories:

- Full-length submissions: Interested experts and researchers may submit a full-length original and previously un-published conceptual or empirical research manuscript for review and decision. Ac- cepted papers will be published in the conference proceedings.

- Research-in-progress submissions: Interested individual researchers who are engaged in postgraduate studies or occupied in research projects for certain enterprises are urged to submit their research plans or projects in progress outcomes.

- Abstracts: All the above submitters of papers should provide and present abstracts of their papers of no more than 100 words indicating the key words to be utilized in the intended papers.

- Paper manuscript Guidelines:

- Manuscripts of full length and in-progress papers should not exceed 15 pages, around 10000 - 15000 words.

- Papers must be accompanied by a separate cover letter with every

author (s) names, address, phone and fax numbers, e-mail, full

affiliation, the track or theme to which it is submitted. All

correspondences will be sent to the first author unless otherwise specified.

- Submitters must provide their e-mail address where the Acknowledgement will be forwarded.

- The paper manuscript must contain, in its first 
page, the paper title,

the author (names, an abstract and a list of keywords.

V. Format for the Proposed and full paper:

- Paper A4 size.

- Margins must be 1 inch.

- Font Face must be "times New Roman" for the Papers written in English, and must be "Simplified Arabic" for the Papers written in Arabic language.

- Font size must be 14 point (Except Paper Title is 18 point and bold).

- Text must be single-spaced.

- Subheadings must be 16 points and bold.

- Must include an ABSTRACT (80 - 150 words in length).

- Submission all proposals and papers must be submitted to the following e-mail address of the organizer of the conference.

- All submitted papers must be electronically in MS Word and submitted electronically.

- The submitted papers must be carefully checked for correct grammar, and spelling.

- Submitters should use the Conference President e-mail addresses

[hadimm1964@hotmail.com],[esisact_08@yahoo.com]and [melhadi@yalla.com].

- All submissions will undergo a double-blind peer review by at least three external scholars reviewers.

VI. Preliminary Deadlines Dates for Abstracts and Reports:

Abstracts/Full-Text Paper Submission Deadline

March 1, 2018

Final Deadline of Paper Reports Submission

March 15, 2018

Notification of Acceptance/Rejection March 25, 2018

\section{Conference Participants and Fees:}

All conference participants, including presenters and attendees must register for the conference, and are urged to pay the appropriate voluntary registration fee as follows: o Interested individuals 100 Egyptian pounds for each Egyptian participant and 300 US\$ for nonEgyptian citizens.

o Faculty staff and trainers 50 Egyptian pounds for each person.

o ESISACT and other Egyptian non-governmental organization members 50 Egyptian pounds for each.

- University and higher institution students 50 Egyptian pounds each.

o Presenters and submitters of research papers and presentation reports 300 Egyptian pounds for each paper or report for Egyptians, and 300 US \$ for foreigners.

- Organizations, institutions, companies and banks membership for 3 persons 300 Egyptian pounds for each organization.

o Organizations, institutions, companies and banks sponsoring the

Conference at least 5000 Egyptian pounds for each at least.

VIII. Conference Sponsor and Exhibitor Opportunities:

The Conference offers the opportunity to become a conference sponsor or exhibitor. To participate as a sponsor or exhibitor, therefor we invite organizations interested to offer their wildlings to share and support the convening of this crucial conference.

\section{CONTACTS:}

Prof. Mohamed M. El Hadi, President of (ESISACT).

Mil Address (38 Syria St., Mohandessin, Giza 12411, EGYPT)

Tel/ Fax (+202) 37613582 ; Mobile: 0100-1411027

E-Mail: melhadi@yalla.com, esisact_08@ yahoo.com, hadimm1964@hotmail.com, mohamed.m.elhadi@gmail.com, \& mohamed.elhady@sadatacademy.edu.eg , melhadi@yalla. com

ESISACT: http://www.esisact.org.eg \& http://www. facebook.com/groups/esisact 